\title{
ESTUDO DA MORBIMORTALIDADE EM PACIENTES COM TRAUMA PANCREÁTICO
}

\author{
Morbimortality in patients with pancreatic trauma
}

\author{
Olival Cirilo Lucena da FONSECA-NETO, Carlos Eduardo Caiado ANUNCIAÇÃO, Antônio Lopes de MIRANDA
}

ABCDDV/517

Fonseca-Neto OCL, Anunciação CEC, Miranda AL. Estudo da morbimortalidade em pacientes com trauma pancreático. ABCD Arq Bras Cir Dig 2007; 20(1):8-11.

RESUMO - Racional - A lesão pancreática é pouco freqüente após traumas abdominais fechados ou penetrantes, e tem sido relatada entre 0,2 a $12 \%$ dos traumas abdominais fechados graves e em cinco a 7\% dos traumas penetrantes. A maioria das lesões pancreáticas ocorre em homens jovens e está associada a alta incidência de lesões a órgãos adjacentes e estruturas vasculares importantes. Objetivo - Avaliar a morbimortalidade dos pacientes com trauma pancreático, o manuseio aplicado a esses pacientes e sua evolução. Métodos - Estudo prospectivo realizado em pacientes admitidos em unidade de trauma. Treze adultos (>13 anos) foram estratificados em graves e não-graves de acordo com critérios clínicos no momento da identificação do início do quadro e de acordo com os critérios de Baltazar (A, B, C, D e E). O tipo de trauma externo (aberto ou fechado), a classificação do trauma pancreático, números de órgãos acometidos pelo trauma, número de reoperações, o tipo de suporte nutricional e o tempo de permanência hospitalar também foram analisados. A presença de síndrome compartimental abdominal e a necessidade de fechamento temporário foram estudados e comparados com a morbimortalidade nos pacientes. Resultados - Todos os pacientes eram do sexo masculino com a idade média de 28,6 anos (13 a 60 anos) e apresentaram pancreatite traumática no pós-operatório. Em sete, o ferimento era penetrante por projétil de arma de fogo; em três, abdominal fechado; em dois, por arma branca; em um, grande queimado ( $>50 \%$ da área corporal). Quanto à classificação do trauma, os graus I e II ocorreram em 38,46; o grau III,15,38 \% e o grau IV, 7,7 \% . Foram realizadas drenagens da loja pancreática, hemostasia da lesão e pancreatectomia distal com esplenectomia associado à drenagem cavitária Foram classificados como não-grave, oito pacientes. Dentro dos critérios de Baltazar a predominância foi o grau D e C (quatro pacientes, cada). Não houve grau A e apenas um foi E. Apenas três pacientes não foram reoperados (média de 1,7 cirurgias por paciente). Em 46,15\%, ocorreu hipertensão intra-abdominal sendo necessário colocação de bolsa de Bogotá. O tempo de internamento variou de 30 à 365 dias (média = 53,4 dias). Ocorreu apenas um óbito. Conclusão - A pancreatite traumática é de elevada morbimortalidade, e uma das complicações mais importantes relacionada ao trauma pancreático, sendo o seu diagnóstico precoce junto com o acompanhamento multidisciplinar intensivo, o fator que poderá interferir favoravelmente na evolução desses pacientes.

DESCRITORES - Pancreatite. Pâncreas, lesões.

\section{INTRODUÇÃO}

O primeiro relato de trauma pancreático foi descrito por Travers, em 1827, e desde então séries de artigos vêm sendo publicadas abordando esse tema. A lesão pancreática é pouco freqüente após traumas abdominais fechados ou penetrantes, e tem sido relatada entre $0,2 \% \mathrm{e}$ $12 \%$ dos traumas abdominais fechados graves e entre $5 \%$ e 7\% dos traumas penetrantes. A maioria das lesões pancreáticas ocorre em homens jovens e está associada com alta incidência de lesões a órgãos adjacentes e estruturas vasculares importantes ${ }^{5}$.

Em relação às feridas abdominais, o mecanismo da lesão é dependente do agente da agressão, arma branca ou projétil de arma de fogo. Os objetos contundentes produzem o trauma diretamente no órgão, tendo como porta de entrada o abdômen superior. Quanto aos traumatismos resultantes por projétil de arma de fogo, a lesão pode ser conseqüente ao impacto direto, ou provocada por ondas de choque. Ainda em relação às feridas por arma de fogo, a lesão resultante é dependente do calibre da arma, da

Trabalho realizado no Serviço de Cirurgia Geral e do Trauma do Hospital da Restauração - SUS - Recife, PE, Brasil.

Endereço para correspondência: Olival Cirilo Lucena Fonseca Neto, e-mail: olivalneto@globo.com velocidade do projétil e se o mesmo foi desviado antes de atingir o alvo. Os traumatismos abdominais abertos são responsáveis por dois terços dos traumatismos do pâncreas ${ }^{3}$.

Nas contusões abdominais o mecanismo mais comum que produz lesão, resulta da compressão do corpo pancreático contra o corpo vertebral produzindo fratura do órgão, associado à lesão duodenal ou não. Outro mecanismo que pode levar ao trauma pancreático é a desaceleração súbita na região periampular podendo levar à desinserção dos elementos vasculares, do colédoco e da própria cabeça de pâncreas ${ }^{8}$.

Dentre as várias classificações existentes para graduar a lesão pancreática, a mais comumente utilizada é a da Associação Americana para a Cirurgia do Trauma (AAST) ${ }^{1}$. Ela freqüentemente é usada porque enfoca tanto a localização anatômica e a extensão da lesão, quanto à condição do ducto pancreático. A graduação adequada utilizando as definições da AAST ajuda a determinar o tratamento a ser realizado de acordo com o grau da lesão ${ }^{4}$.

$\mathrm{O}$ tratamento do trauma pancreático varia desde estratégias não cirúrgicas até ressecções cirúrgicas amplas ou mesmo controle de danos, dependendo da gravidade e do local da lesão ${ }^{10}$. 
A pancreatite aguda (PA) é uma das complicações mais temerosas pós-trauma pancreático. Dentre as pancreatites agudas, ela representa cerca de $10 \%$, porém, são mais graves ${ }^{6}$ Uma das formas de caracterizar a gravidade da pancreatite aguda é a desenvolvida por Balthazar ${ }^{1}$ que analisa o valor prognóstico da tomografia computadorizada realizada mediante contraste intravenoso com infusão em bolo. Na fase inicial da PA, eles idealizaram um índice de gravidade com base na avaliação dos achados tomográficos (Tabela 1).

TABELA 1 - Critérios tomográficos de Balthazar ${ }^{1}$

\begin{tabular}{|c|c|c|}
\hline \multicolumn{2}{|l|}{ Graus de pancreatite } & Pontos \\
\hline \multicolumn{2}{|l|}{ - aumento do pâncreas } & 1 \\
\hline \multicolumn{2}{|c|}{ - inflamação pancreática e peripancreática } & 2 \\
\hline \multicolumn{2}{|l|}{ - uma coleção líquida } & 3 \\
\hline \multicolumn{2}{|c|}{ - duas ou mais coleções líquidas } & 4 \\
\hline \multicolumn{3}{|c|}{ Graus de necrose pancreática } \\
\hline \multicolumn{2}{|c|}{ - necrose de $1 / 3$ do pâncreas } & 2 \\
\hline \multicolumn{2}{|c|}{ - necrose de $1 / 2$ do pâncreas } & 4 \\
\hline \multicolumn{2}{|c|}{ - necrose de mais de $1 / 2$ do pâncreas } & 6 \\
\hline Índice ( $n^{0}$ de pontos) & Complicações (\%) & Mortalidade (\%) \\
\hline 0 a 1 (A) & 0 & 0 \\
\hline 2 a 3 (B) & 8 & 3 \\
\hline 4 a $6(C)$ & 35 & 6 \\
\hline 7 a 8 (D) & 92 & 17 \\
\hline
\end{tabular}

Altas morbidade e mortalidade estão associadas ao trauma pancreático, com taxas relatadas de 30 a $50 \%$ e de 10 a $32 \%$, respectivamente, tornando imperativo o diagnóstico precoce e o tratamento acurado?

Tendo em vista a relativa escassez de trabalhos evidenciando a conduta no trauma pancreático e suas principais complicações e manejo, este estudo objetiva demonstrar a experiência emm uma série de casos na identificação e manuseio dos pacientes com trauma pancreático e sua evolução.

\section{MÉTODOS}

Este é um estudo prospectivo realizado em pacientes admitidos na unidade de trauma da emergência do Hospital da Restauração, Recife, PE, Brasil, entre janeiro de 2004 e janeiro de 2006. Eram todos adultos ( $>13$ anos) e foram acompanhados pelo mesmo grupo até a sua alta ou óbito.

Foram estratificados em graves e não-graves de acordo com critérios clínicos no momento da identificação do início do quadro e de acordo com os critérios de Balthazar (Tabela 1).

O tipo de trauma externo (aberto ou fechado), a classificação do trauma pancreático, números de órgãos acometidos pelo trauma, número de reoperações, o tipo de suporte nutricional e o tempo de permanência hospitalar também foram analisados.

A presença de síndrome compartimental abdominal e a necessidade de fechamento temporário foram estudados e comparados com a morbimortalidade nos pacientes.

\section{RESULTADOS}

Foram acompanhados treze pacientes com trauma pancreático nesse período, todos eram do sexo masculino com a idade média de 28,6 anos (13 à 60 anos) e apresentaram pancreatite traumática no pós-operatório. Encontrou-se em sete pacientes, ferimento penetrante por projétil de arma de fogo (PAF); em três, trauma abdominal fechado (TAF); em dois, ferimento penetrante por arma branca (FAB) (Gráfico1). Um dos casos, foi grande queimado (> 50\% da área corporal).

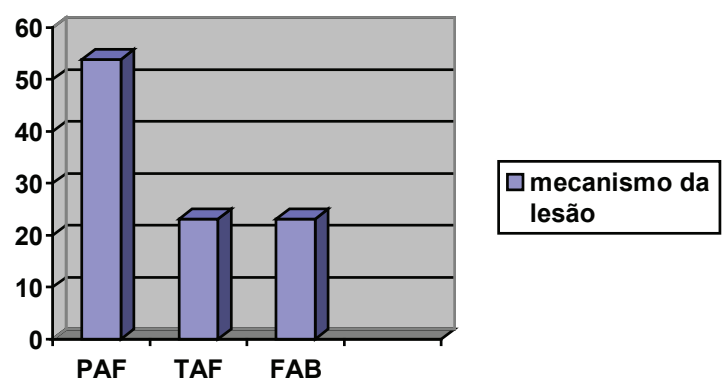

PAF: ferimento penetrante por projétil de arma de fogo; TAF: trauma abdominal fechado; FAB:ferimento penetrante por arma branca

GRÁFICO 1 - Mecanismo da lesão pancreática

Não ocorreu nenhum paciente classificado com trauma grau V. O grau I se fez presente em $38,46 \%$; da mesma forma o grau II, $38,46 \%$; o grau III, $15,38 \%$ e o grau IV, $7,7 \%$ (Gráfico 2).

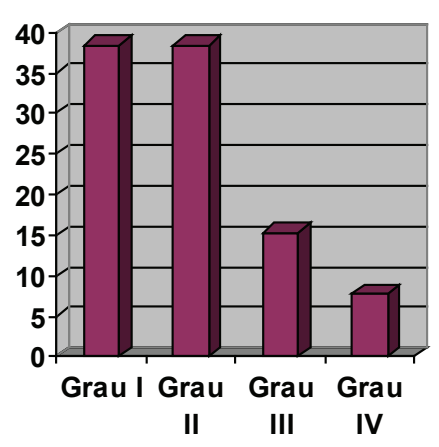

Classificação do trauma pancreatico

GRÁFICO 2 - Classificação do trauma pancreático

Dos pacientes com lesão grau I, dois foram submetidos à drenagem de loja pancreática apenas e outros três só tiveram seu diagnóstico de trauma pancreático quando foram reoperados (diagnóstico de pancreatite transoperatório); já os classificados no grau II, foram submetidos à hemostasia da lesão (sutura do parênquima ou ligadura de vasos) quando necessário e drenagem cavitária; nos pacientes com grau III, foi realizada pancreatectomia distal com esplenectomia associada à drenagem cavitária e no paciente com grau IV foi apenas realizado sutura da lesão sangrante e drenagem cavitária, pois ele apresentava-se muito grave, em choque hipovolêmico (Tabela 2). Todas as drenagens realizadas foram em sistema aberto com bolsa de colostomia. 
TABELA 2 - Opções terapêuticas conforme o grau da lesão pancreática

\begin{tabular}{lccccccc}
\hline $\begin{array}{c}\text { GRAU } \\
\text { Forma de tratamento: }\end{array}$ & I & II & III & IV & V & $\begin{array}{c}\text { TO- } \\
\text { TAL }\end{array}$ & $\%$ \\
\hline - Simples drenagem & 2 & 5 & 2 & 1 & - & 10 & 35,71 \\
- Sutura pancreática & - & 3 & 2 & 1 & - & 6 & 21,42 \\
- Ressecção pancreática & - & - & 2 & & - & 2 & 7,14 \\
\hline
\end{tabular}

Foram classificados como não-grave pelos critérios clínicos (sem uso de vasopressor e ausência de insuficiência renal) oito pacientes. Dentro dos critérios de Balthazar a predominância foi o grau $\mathrm{D}$ e C (quatro pacientes, cada). Não houve grau A e apenas um foi E (Tabela 3).

TABELA 3 - Classificação de Balthazar dos pacientes

\begin{tabular}{cc}
\hline Critérios de Balthazar & $\mathbf{N}^{\mathbf{0}}$ de pacientes em \% \\
\hline A & ----- \\
B & 30,76 \\
C & 30,76 \\
D & 30,76 \\
E & 7,69 \\
\hline
\end{tabular}

O número de órgãos acometidos variou de 1 a 5 (média $=3$ ) por paciente. Os lesados com maior freqüência foram cólon, estômago e fígado $(53,8 \%, 38,4 \%$ e $38,4 \%)$ respectivamente (Gráfico 3). Em relação ao manejo nutricional, a nutrição parenteral foi a escolha em $46,1 \%$ dos pacientes e apresentou como complicação maior a infecção do sítio de punção em dois casos. Não houve sepse originária do cateter. A nutrição enteral ocorreu em 53,8\% e a jejunostomia foi a preferência na via de acesso $(57,1 \%)$ que apresentou como complicação o vazamento da estomia em um caso.

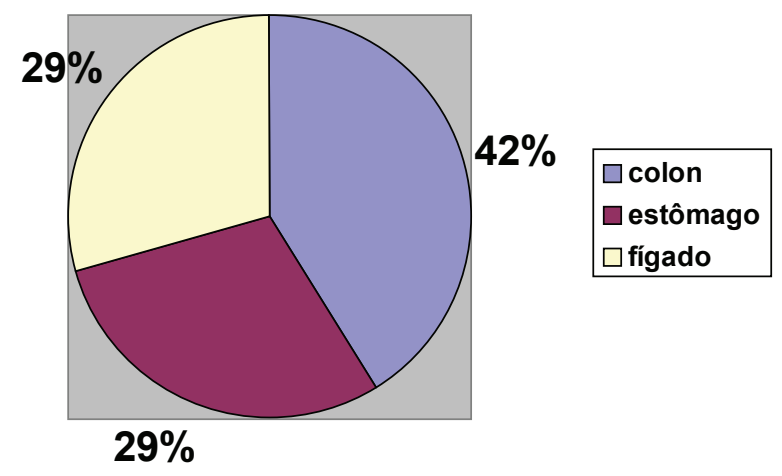

GRÁFICO 3 - Órgãos mais lesados associados ao trauma pancreático

Apenas três pacientes não foram reoperados (média de 1,7 operações por paciente). Em $46,15 \%$ dos pacientes ocorreu hipertensão intra-abdominal sendo necessária a colocação de bolsa de Bogotá. O tempo de permanência foi maior naqueles com a nutrição parenteral e variou de 30 à 365 dias (média $=53,4$ dias). Ocorreu apenas um óbito (fazia uso de nutrição parenteral).

\section{DISCUSSÃO}

O trauma pancreático se constitui em desafio para o cirurgião na sala de emergência, seja pelo desafio diagnóstico, seja pela sua complexidade terapêutica. Para a escolha da melhor opção de tratamento deve-se levar em conta o estado do paciente, o grau de lesão e o conhecimento técnico e habilidade cirúrgica do cirurgião.

Neste estudo o ferimento penetrante por projétil de arma de fogo foi o mais freqüente, seguido pelo trauma fechado e por último por arma branca. Estes dados variam de acordo com o país estudado e as condições de desenvolvimento da região. Esta é a razão de haver discrepâncias entre as séries apresentadas $^{2,8}$. As queimaduras não apresentam lesão pancreática com freqüência, e é o que foi verificado nesta série onde somente um dos casos foi grande queimado.

A drenagem de loja pancreática apenas é procedimento possível nos pequenos traumas e nos pacientes que estão em grave estado geral onde procedimentos cirúrgicos mais abrangentes possam ser contraindicados. É difícil padronizar a operação a ser feita em função da multiplicidade de lesões ${ }^{10}$.

A classificação de Balthazar ${ }^{1}$ é bom parâmetro de prognóstico e mostrou correspondência e correlação neste estudo dos critérios mais avançados com os resultados de maior taxa de complicações. Por ser simples de aplicação ela deve ser usada na rotina desses pacientes.

Consoante com a literatura, o número médio de órgãos associados foi de três por paciente, com incidência maior no cólon, estômago e fígado.

A nutrição parenteral foi utilizada em quase metade dos pacientes e não apresentou complicação importante. Por outro lado, o benefício que ela representou, estimula sua utilização para repouso do pâncreas até a estabilidade do quadro de maneira rotineira nos grandes traumas quando não é possível a utilização de dieta enteral.

A nutrição enteral ocorreu em 53,8\% desta série e a jejunostomia foi a preferência na via de acesso que apresentou não apresentou complicações relevantes.

O manuseio nutricional dos pacientes com a pancreatite pós-traumática quanto ao tipo e forma, deve ser individualizado e otimizado para melhorar a adaptação hipermetabólica com os nutrientes oferecidos; e assim, melhorar o prognóstico desses pacientes com diminuição do tempo de permanência hospitalar e do custo gasto com a alta complexidade no tratamento.

\section{CONCLUSÕES}

A pancreatite traumática é de elevada morbimortalidade, e representa uma das complicações mais importantes relacionada ao trauma pancreático, sendo o seu diagnóstico precoce, junto com o acompanhamento multidisciplinar intensivo, o fator que poderá interferir favoravelmente na evolução desses pacientes. 
Fonseca Neto OCL, Anunciação CEC, Miranda AL. Morbimortality in patients with pancreatic trauma. ABCD Arq Bras Cir Dig 2007;20(1):8-11.

ABSTRACT - Background - Pancreatic lesion is uncommon after closed or penetrating abdominal trauma, being related between 0,2 to $12 \%$ in severe closed abdominal traumas and in 5 to $7 \%$ of penetrating traumas. The majority of pancreatic lesions occur in young men and is associated with an increased incidence of trauma in adjacent organs and major vascular structures. Aim - To evaluate morbimortality rates, evolution and handling of patients with pancreatic trauma. Methods - A prospective study of patients admitted to the trauma unit was made. Thirteen adults ( $>13$ years-old) were divided into two groups one composed of severe and the other composed of non-severe patients according to clinical criterion in the moment of identification of trauma using the Balthazars' criteria (A, B, C, D and E). The type of external trauma (opened or closed), classification of pancreatic trauma, number of organs reached by trauma, number of reoperations, type of nutritional support and period of time in the hospital were also analyzed. Presence of compartmental abdominal syndrome and necessity to close trauma temporarily were studied and compared to morbimortality in patients. Results - All patients were male, with an average age of 28,6 years (13 to 60 years of age), presenting postoperatory traumatic pancreatitis. Seven patients, showed penetrating wounds due to firearms; three had closed abdomen; two had knife wounds and one had severe burns (> $50 \%$ of body area). Trauma classification showed, I and II degree in 38,46\%; III degree in $15,38 \%$ and IV degree in 7,7\% of patients. Pancreatic drainage, lesion hemostasia and distal pacreactomy with splenectomy associated with cavity drainage were performed. Eight patients were classified as non-severe. According to Balthazars' criteria, grades D and C were predominant, with four patients each. There were no grade A patients and only one was grade E. Only three patients were reoperated (average of 1,7 surgeries per patients). In 46,15\% intra-abdominal hypertension occurred, making it necessary to apply the Bogota bag procedure. The period of admission varied between 30 to 365 days (average of 53,4 days). Only one death was registered. Conclusions - Pancreatitis due to trauma has elevated morbimortality rates and is one of the most significant complications related to pancreatic trauma. Consequently early diagnoses along with intensive multidisciplinary follow-up are important and determinant factors which directly interfere in a favorable evolution of these patients.

HEADINGS - Pancreatitis. Pancreas, injury. Mortality.

\section{REFERÊNCIAS}

1. Díaz, P, Saenz R,; Watkins, G. Godoy AP, et al. Criterios pronóstico de Ranson y Balthazar y aplicabilidad de criterios de Atlanta 1992 en pancreatitis aguda grave / Ranson and Balthazar prognostic criteries and aplicability of Atlanta 1992 criteries in severe acute pancreatitis Gastroenterol. latinoam;9(2):150-57, sept. 1998.

2. Moore EE, Cogbill TH, MA Malangoni, GJ Jurkovich. Organ injury scaling, II: Pancreas, duodenum, small bowel, colon, and rectum. J. Trauma. 1990 Nov 30(11):1427-9.

3. Freire E, Ribeiro F. A.S, Manso J. E. F. Trauma de Pâncreas Trauma: a doença dos séculos. $1^{\mathrm{a}}$ ed., Rio de Janeiro: Atheneu; 2001 Vol II cap. 117 p. 1569 76

4. Mainwaring, JE. Trauma duodeno pancreático / Duodenum pancreato trauma Uiv. med.; 28(1):45-50, ene. 1986.

5. Ribeiro F A, Maia F J, Souza, A . Trauma de Pâncreas. In: Clínica Cirúrgica - Fundamentos Teóricos e Práticos. $1^{\mathrm{a}}$ ed., Rio de Janeiro: Atheneu; 2000. cap. 38 p. 378- 374.
6. Santos JS, Junior EE, Scarpelini S \& Sankarankutty AK. Pancreatite aguda: atualização de conceitos e condutas. Medicina, Ribeirão Preto, 36: 266-82, abr. /dez. 2003.

7. Schwaner JC, Rivas FB, Cancino AN, et al. Pancreatitis Aguda: Indice de severidad en TC evaluación de complicaciones y hospitalizacion Rev. chil. radiol. v.9 n.4 Santiago 2003.

8. Stalhschmidt CMF, Formighieri B, Lubachevski FL. Controle de danos no trauma abdominal e lesões associadas: Experiência de cinco anos em um serviço de emergência. Rev. Col. Bras. Cir.Ago 2006, vol.33, no.4, p.215-9.

9. Von Bahten, Luiz Carlos et al. Fatores prognósticos em pacientes submetidos à cirurgia por trauma pancreático. Rev. Col. Bras. Cir. Out 2004, vol.31, no.5, p.332-7.

10. Schein M. Current management of trauma to the pancreas. Br J Surg. 1992 Jul;79(7):717-8.

Conflito de interesse: não há

Fonte financiadora: não há

Recebido para publicação em: 23/09/2006

Aceito para publicação em: 11/12/2006 